\title{
Sleep Monitoring Tools at Home and in the Hospital: Bridging Quantified Self and Clinical Sleep Research
}

\author{
Bert Vandenberghe, David Geerts \\ CUO $\mid$ Social Spaces \\ iMinds - KU Leuven \\ Leuven, Belgium \\ firstname.lastname@soc.kuleuven.be
}

\begin{abstract}
The quantified self movement suggests solutions for diverse long-term measurements, including sleep monitoring. However, those solutions do not seem to meet the challenges facing clinical sleep research. Where efforts in the past to describe design frameworks for sleep monitoring tools focused on the sleeper as user, we start from the sleep clinician to find out how sleep monitoring tools can be meaningful in clinical settings. Based on observations in hospital-based sleep centers performing traditional and ambulatory sleep studies, we describe current practices and look at the effect when measurements leave the hospital. We summarize design recommendations for sleep monitoring tools, suitable in and outside the hospital, from the sleep clinician's perspective. Furthermore, we discuss a future for sleep research where quantified self tools and approaches expand clinical sleep research. This would allow hospital-based sleep centers to deploy current practices in a targeted, meaningful, and accountable way.
\end{abstract}

Keywords-Design; Health care; Sleep center; Sleep monitoring; Personal informatics; Quantified self

\section{INTRODUCTION}

Using quantified self approaches, people choose to keep an eye on various aspects of their life, e.g. their sleep behavior. These self-initiated methods allow people to monitor themselves for a longer period of time using unobtrusive tools. The collected data can contribute to self-awareness and selfknowledge through self-reflection. But despite the abundance of smartphone apps and devices, quantified self experiments stay non-binding and unreliable from a clinical perspective [7], which is troublesome when people having severe sleep disorders start using these tools instead of going to a specialized sleep center.

Given the success of easily accessible sleep monitoring apps and devices, there is a need for new clinical validated sleep monitoring tools to expand the current practices, that can lead to more efficient diagnosis and treatment of sleep related disorders [12]. Today, most sleep research is being performed in specialized sleep centers, often associated with hospitals. Here, sleep clinicians rely on polysomnography (PSG) to examine patients during sleep. PSG is an intensive, extensive, and therefore expensive technique, limiting the capacity of sleep centers today. This leaves a growing group of patients undiagnosed [21], which might turn to unreliable self- monitoring tools. Also, PSG is considered as overkill for screening purposes, and not suitable for prolonged usage, e.g. during follow-up.

In the quantified self philosophy, the quantified selfer makes the conscious choice to perform the measurements on him, while in the hospital the clinician decides to perform the measurements on the patients. But although clinical studies and quantified self measurements have an opposite approach, they don't have to exclude each other. In a future where the quantified self movement expands clinical sleep research by extending monitoring in place and time, long-term measurements in a home environment could support screening and medical treatment as well as solid self-knowledge. Sleep monitoring could then be performed on prescription or on a person's own initiative. Sleep centers can then focus on treatment, resulting in a more effective use of the limited capacity at sleep centers. People or patients could also have more control on the measurements in their own environment, whether or not guided by clinicians depending on the patient's preference.

The change from centralized sleep studies to decentralized screening of sleep disorders and sleep monitoring during follow-up may be obvious from the perspective of the quantified self, but it is a disruptive change from a clinical point of view. Remote measurements in unknown environments are unfamiliar territory for the sleep clinician, who is used to have full control over the nearby equipment. By bridging the gap between the home and the hospital, some challenges for the design of the sleep monitoring tools emerge.

This paper addresses these challenges by describing considerations and recommendations for the design of sleep monitoring tools, founded on observations in hospital-based sleep centers. With this approach, we hope to provide a clear view on the clinician's perspective during the design of futureproof sleep monitoring tools. These tools must at all times support the sleep clinician in their main task: identifying sleep related disorders, and must be suitable to be used in the hospital and at home.

Including the sleep professional in the design of sleep monitoring tools, an approach which to our knowledge has not been done before, can make the tools fit in a clinical setting. Sleep professionals can then deliver a major contribution to the 
interpretation of complex sleep patterns, and to the clinical validation of these tools. These validated sleep monitoring tools can then give meaningful output to the quantified selfer, and deliver meaningful input to the sleep clinician. The sleep professional can choose to use the tools in his turn as well in hospital-based sleep studies or in ambulatory sleep research, and transcend borders between the home and the hospital. This could solve capacity problems in the hospital, and current techniques could be used in a more targeted and efficient way.

\section{BACKGROUND}

The goal of a clinical sleep study is to identify sleep related problems such as snoring, sleep apnea (short repetitive breathing stops during sleep), insomnia (inability to sleep), limb movement disorders, and parasomnia (undesirable events or experiences during sleep, falling asleep, or waking up) [1]. Since the causes of these problems can be diverse, sleep research is a multidisciplinary activity. In general, the following specialisms are involved in sleep research: ENT (ear nose throat), neurology, pneumology, and psychiatry. Other specialisms that can be involved as well are: cardiology, neonatology, nursing, and pediatrics. This makes sleep study, and especially the interpretation of the cause and effect of sleep related problems a complex matter.

The gold standard in sleep research is PSG. This technique measures the sleep quantity and structure, and how many times the sleep is disturbed. Next to that, some physiological processes such as heart rate or ventilation (air flow during inhalation or exhalation) are monitored that can affect sleep. By examining the sleep quality and the physiological processes, relations between the two can become clear and a cause for the sleep disorder(s) can be found. To do the measurements and monitoring, multiple state-of-the-art sensors are stuck on the patient's head, face, and body while sleeping. To process the information, trained personnel are required [21]. Altogether, PSG is a complete but expensive study, and therefore not appropriate to repeat multiple times during a longer period.

Next to PSG, some alternative sleep research methods are available. PG (polygraphy) is a stripped down version of PSG, in which no information about the sleep structure is measured or monitored [21]. This makes PG less inconvenient, less expensive, but also less complete than PSG. With PG only, not all sleep disorders can be identified. PG is based on the same theories as PSG and still requires wired on body measurements. Some new methods such as actigraphy [14] are being developed to allow wireless and/or off body measurements, which are less intrusive for the patient. These methods are also cheaper, allowing long-term measurements.

In theory, the three methods described above can be performed in a clinical or ambulatory setting, but in practice PSG is mostly performed in a hospital because of the amount of expensive equipment that is required [21]. Besides, when the patient sleeps in the hospital, a trained nurse or technician can attend the study during a clinical PSG to provide extra information during the night and fix problems with the equipment in case of loose or broken connections. Wireless and/or off body measurements in a home environment can be less stable, because breakdowns only become clear afterwards. So there is a tradeoff between expensive but guaranteed measurements in the hospital versus cheaper but unsure measurements in a home environment.

\section{RELATED WORK}

We start with some examples of how the technical and medical domains are looking for new techniques to measure and monitor sleep, and the resulting opportunities for the domain of HCI. Also, we illustrate how the quantified self movement drives the work on new sleep monitoring tools. We conclude this section with some commercial successes and failures in the field of sleep.

There is already a lot of work done in the technical and medical domains that suggest alternatives for the expensive PSG in the hospital or at home. Zhao et al. [25] describe a method where only the ECG signal is used to determine sleep quality. This method eliminates a lot of sensors, making the measurements less intrusive for the patient. This system is promising, but is designed for a specific multimedia application rather than a clinical one. Other work, such as SNORES, demonstrates the use of wireless sensor networks in a home environment to screen patients, and refer them to a hospital in case of severe problems [11]. Shambroom et al. describe the validation of an easy to use and accurate wireless system for measuring sleep by comparing the new technology with PSG and actigraphy. Their findings prove that alternative methods could be accurate [20]. These solutions mainly aim to make sleep monitoring less intrusive for the sleeper. Although some of these solutions bring clinical sleep research closer to the home, there is little involvement of the sleep professional in the design of the tools. Therefore, these solutions don't always meet the needs of the sleep professional and stay non-binding from a clinical perspective. These tools are nice-to-have, but less useful for the clinician.

The ongoing technical and medical developments lead to new opportunities in the domain of sleep for the HCI community, which are already well documented. Koreshoff et al. look at the Internet of Things (IoT) for trends, gaps, and opportunities for HCI, including the domain of sleep [13]. Aliakseyeu et al. provide a rich understanding of sleep and its social aspect, to highlight the opportunities for interaction design research around sleep [2]. Choe et al. state that, in contrast to supporting a healthy diet and exercise, the support of healthy sleep behavior is understudied in HCI [7]. They provide design considerations for tools that improve sleep behavior, based on a literature review and contextual interviews with experts and patients. While they identified a gap for sleep monitoring tools that serve diagnosis and treatment, the design considerations focused on the sleeper as user. By focusing on the sleeper only, new tools risk not fitting in a clinical environment, making it hard to cross borders between the home and the hospital.

The quantified self movement drives some of the existing work on sleep, e.g. by using a mobile phone application for everyday sleep monitoring. In one study, using a smartphone app, persons are asked to respond to a tone at regular intervals. If the person is not responding, then he or is sleeping [15]. In 
another study a smartphone is also used to remind people of good sleep hygiene by showing them tips on an active wallpaper [4]. These solutions focus on self-management of sleep hygiene. Several authors state that by measuring sleep, people become self-aware. One example can be found in [8], where a persuasive app is designed to help people achieving their sleep related goals. By self-reflection and self-treatment, people can improve their sleep behavior themselves. In practice, however, we see that interpretation of complex sleep patterns can be difficult for an uninitiated person, even with the aid of advanced software algorithms. As described in [16], people often can't relate cause and effect of certain problems, because they don't understand the broader context. This prevents self-reflection from being successful. Also, as the authors describe, existing tools lack understanding of people's self-reflecting needs, which makes a correct interpretation even harder. Pirzadeh et al. [18] provide a deeper understanding of the process of self-reflection, and argue that enough information and a proper interpretation is required for successful self-reflection, otherwise the effect on behavior change is limited. As a sleep professional can assist the sleeper to make a correct interpretation of his sleep by providing insight in the monitored signals, it can be helpful to consider the sleep professionals as an end-user as well, and involve them in the design of sleep monitoring tools.

Paalasmaa et al. describe the use of an unobtrusive online monitoring system. Here, a piezoelectric sensor is placed under the mattress topper and sends data to a web server for analysis. Users can also augment the recorded data with tags (e.g. stress, alcohol, or exercise). The authors suggest using this new method for a few weeks, after which a doctor can interpret the data and decide whether the patient needs a full PSG or not [17].

Gartenberg et al. go one step further, and describe an iPhone app for diagnosis and treatment of insomnia. Here, realtime tracking is used together with retrospective editing to ensure high quality data [10]. Zhang et al. describe a smart pillow that detects sleep apnea, and automatically adjusts itself in order to resolve it [24].

Despite these efforts, we see that medical validation is hard for new technologies. Wright explains this phenomenon by pointing at the conservative nature of medicine, but also warns for the danger of misinterpretations by patients [22]. Involving clinicians in the design of tools for use at home can be beneficial for both sides. Kelly et al. describe the recent developments in home sleep monitoring devices. Although they have some comments on the current costly and timeconsuming validation studies, they also express the need for them. These validation studies can improve the understanding of the capabilities and limitations of the sleep monitoring tools for home usage, in order to maximize their utility [12].

Finally, in the past ten years, some commercial products were launched in the domain of sleep. The Philips Wakeup Light $^{1}$ lets people wake up in a more natural way using light and sounds, to mimic sunrise and nature. The Fitbit One ${ }^{2}$ tracks

http://www.usa.philips.com/c-m-li/light-therapy/wake-up-light http://www.fitbit.com/one daily activity, including sleep. The Beddit ${ }^{3}$ system combines the data of a sensor in the bed with an app to provide people feedback on their sleep, and to improve it. The Withings Aura ${ }^{4}$ combines a bed sensor with an app and a bedside device, to monitor and even impact sleep by emitting light and sound. This proliferation of products proves that there is a market for such systems. However, one of the quantified self pioneers, Zeo (an alarm clock that measured sleep stages, in order to wake people only during light sleep), went out of business somewhere in 2013 [9]. This event followed that of another sleep tracker Wakemate (a device based on actigraphy, that was able to wake people only during light sleep), running out of cash mid 2012 [6].

We can conclude that some work on sleep is already being done in the field of HCI, and there were some varying degrees of commercial success for consumer products. Although the sleep professional has been taken into account in some work, e.g. to increase the efficiency and to optimize the management of a sleep center [19], to our knowledge, most work concerning sleep monitoring focuses on the sleeper as user.

In our work, we involve the sleep professional as user in the design of sleep monitoring tools by observing their work and the environment in which they work. This makes the new tools suitable for both a home and a hospital environment, resulting in mutual benefits for both sleep medicine and the quantified self movement by exchanging knowledge. Other domains in healthcare showed that this approach looks promising, e.g. for mental illness [3]. As Yunan et al. describe, bridging the home and the hospital can also help to meet the growing needs in aging populations and to deal with the challenges in developing regions. They highlight the complexity and uniqueness of healthcare practices, and issues within and outside the clinical setting [23]. In this paper, we discuss how this approach could work in the domain of sleep.

\section{Methodology}

We did observations in clinical sleep centers, in order to understand the current practices in clinical sleep research. We decided to focus on PSG, the existing gold standard in sleep research, because the technique is widespread in hospitals. Alternatives of the PSG technique are rare. We selected three sleep centers, in which we observed multiple sleep clinicians. Two of the selected sleep centers were leading sleep centers in Belgium, both conducting a large number of sleep studies in parallel, so we were able to observe sleep research on a large scale, and could observe multiple studies being prepared, performed, and analyzed by different clinicians in one day. As a third sleep center we wanted a center performing ambulatory sleep studies, as this approach already offers a mix between hospital-based and home based sleep monitoring. Because of this requirement, we had to select a sleep center in the Netherlands, since Belgian sleep centers do not perform these kinds of studies (due to reimbursement policies).

We started the observations in a leading sleep center in Belgium with a very rich history in sleep research, associated

http://www.beddit.com

http://www.withings.com/en/aura 
with a university hospital. This multidisciplinary sleep center is operational 24/7 and performs over 4000 sleep studies per year. At this sleep center, patients are welcomed during the afternoon, so they can get used to the environment and sensors before they sleep in the hospital. They get prepared by a technician, who pastes sensors on the patient's head, body, and legs. The patients sleep one night at the hospital, and get the sensors removed by a technician in the morning. Normally, the patient can go home after one night. In the following weeks, technicians and doctors analyze and review the recorded signals - sensor, audio, and video. At the end, the sleep center provides a report with diagnosis and a suggestion for treatment if applicable.

At this first sleep center, we observed the sleep clinicians, their tasks, and the environment in which they perform these tasks, to understand what they do and why they do this. We made detailed notes of the observed actions and conversations. Due to strict rules in the hospital, we were not allowed to make audio or video recordings. During the observation, we asked questions to clarify intents of and strategies behind certain actions. To take into account personal ways of working, four persons were observed doing their work ca. 1 h30 each. These four persons had different roles and responsibilities. We observed the technical head of the sleep center who was responsible for selecting and configuring the equipment at the center, and three nurses or sleep technicians preparing, executing, and analyzing sleep studies. We also interviewed the responsible physician for the medical motives behind sleep research, the analysis process, and the supporting sleep monitoring tools. This sleep center had a rather flat organization, with shared responsibilities among clinicians.

To distinguish hospital procedures from sleep research practices, a second sleep center with similar setup, also associated with a university hospital, was selected to perform an observation. This second sleep center uses the most recent technological equipment, is $24 / 7$ operational, and has the capacity to perform over 3500 sleep studies per year. At this sleep center, sleep studies are performed in the same way. Patients arrive at the sleep center, get prepared, sleep at the hospital, and go home the day after. During the next days, nurses, interns, and specialists analyze and review the recorded data in order to deliver a report with a diagnosis and suggestion for treatment. We interviewed the head of the sleep center and observed three people doing their work, ca. one hour each. These people included the head nurse of the sleep center, one nurse preparing and analyzing studies, and one nurse preparing the studies. We observed a rather hierarchical organization, with multiple levels of responsibility among clinicians.

Finally, to discover the differences between hospital-based and ambulatory sleep research, a third hospital in the Netherlands was contacted for a final observation. At the sleep research unit of the neurology department of the hospital, 800 ambulatory sleep studies per year are being performed. Patients get prepared at the hospital by a sleep technician, they go home and sleep in their bed, and the day after they return to the hospital to get the sensors removed by the technician. In this center we observed one person that prepared and analyzed the studies during one day. In this sleep center, the technicians had more autonomy and could handle more patients per day, because the contact with the patient is much shorter.

In total, we observed eight people with different roles and responsibilities in three different hospitals while preparing, executing, and processing sleep studies in a hospital-based and ambulatory setting. As there are only a few large sleep centers in Belgium performing enough studies to have an elaborated workflow that we could observe, this selection of sleep centers is sufficient to get a clear image of work procedures for sleep monitoring. However, other countries might have different procedures that are not covered by our observations.

The notes were translated into work models and an affinity diagram [5]. The work models focused on the information flow and interaction between people to uncover a general sleep study procedure and some considerations for the design of sleep monitoring tools. The affinity diagram was used to uncover patterns in the interviews and observations that could lead to considerations and recommendations for the design of sleep monitoring tools. These results were consolidated in a detailed report that was presented and discussed with the clinicians (both nurses and physicians) of the first hospital who were observed and interviewed during their work, to make sure we made a correct interpretation of the observed workflow and to validate the findings.

\section{RESULTS}

We describe the current practices in clinical sleep research and take a look at the effects of taking the measurements outside the hospital, by comparing the clinician's workflow when the patient sleeps in the hospital in hospital-based studies and when the patient sleeps at home in ambulatory sleep studies. We learned that if tools fit in the hospital infrastructure and procedures, explain to the patient what is happening when the clinician is not present, give the clinician access to raw data, enrich the recorded data with context, and enables the clinician to share the data with colleagues, they can be useful in the hospital, and at home.

\section{A. Fit in the hospital}

Sleep centers use an extensive range of tools to perform sleep studies today, such as consumables that can only be used once (e.g. body patches, masks, wires, and glue), sensors that are cleaned and reused for every study (e.g. pulse oximeter that measure the oxygen saturation of the patient), devices to record sensor data (e.g. a headbox that connects all sensor wires), systems to store this information, and software (e.g. to analyze the recorded data). This equipment is, or should be, interchangeable following standards such as the EDF+ format ${ }^{5}$.

To be attractive for the clinical sleep research market, new sleep monitoring tools should fit in existing hardware and software, using existing standards. In this way, the new tools could supplement or even supersede current components in the system. While this recommendation might seem self-evident, our observations show that in practice manufacturers don't follow industry standards, limiting the sleep center and its clinicians. This results in a range of tools that don't find

$$
\text { http://www.edfplus.info }
$$


entrance in the hospital, and a proliferation of workarounds. We saw e.g. clinicians using two separated computers next to each other, transferring data via a USB drive, because software that doesn't follow standards is not allowed on the hospital network. As with all hardware and software standards, hospitals have defined procedures and policies for their use. Tools that impose a specific workflow or procedure are hard to use in a hospital environment as well.

Based on these observations, we recommend to let the sleep center or clinicians use the tool as they are used to perform sleep studies, or let the sleep center adapt the workflow to their own. These workflows have a history, and are unlikely to change because of a tool that does not fit in. PSG is here to stay, at least for a while, so new tools should fit the current process. New tools can provide additional information, or be used prior to or after the current studies (e.g. during screening or follow-up). New tools are unlikely to disrupt the established procedures in hospitals.

\section{B. Speak to the patient}

In hospital-based sleep research, the patient arrives in the afternoon. Clinicians welcome the patient, and have the rest of the day to explain the procedure to the patient. In the morning, they wake the patient and they immediately tell the patient whether the recordings were successful. This reassures the patient that the past night, often with uncomfortable sensors, was worth it. The clinicians also explain the upcoming steps (e.g. what the doctors will do with the recorded signals, when
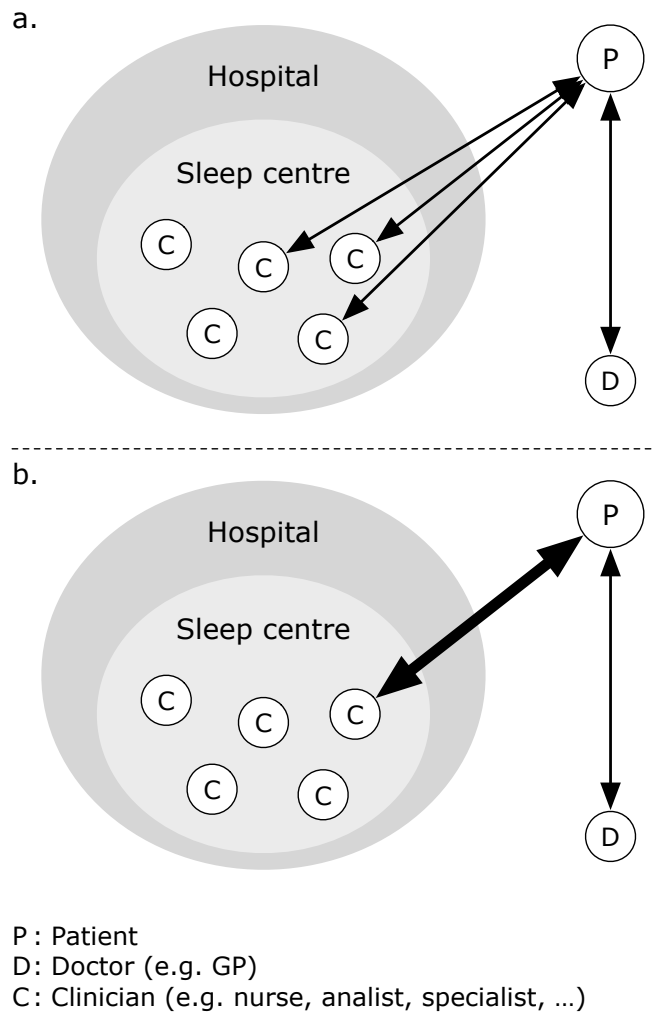

Fig. 1. Communication with the patient is more spread in a hospital-based sleep study (a), than in an ambulatory sleep study (b), both in time and among clinicians. In an ambulatory setting, communication with patients becomes more concentrated and thus crucial. they can expect the results, ...). Several clinicians told us that this process of making the patient understand what is happening is very important factor for compliance (i.e. how well patients tolerate what is happening and follow instructions or prescriptions).

When the sleep study is performed outside the hospital, e.g. in ambulatory sleep research, the contact moments with the patient are reduced to a bare minimum. This makes the remaining communication between clinician and patient very concentrated and crucial, as is illustrated in Fig. 1.

A sleep monitoring tool that can explain to the patient what is happening is helpful regarding compliance. Then the patient can be supported even when the clinician is not present. This can be achieved by visualizing the recorded data, and providing explanation in each step of the process. We therefore recommend that sleep monitoring tools show the patient what is happening, while it is happening.

\section{Also provide raw data}

While processed and visualized data are very useful to explain to the patient what happens for compliance reasons, the clinicians want raw data. Or as one of the observed clinicians puts it:

"There is software on the market with very 'revolutionary' features, but these manufacturers forget we're working in a medical environment. We diagnose patients by comparing and recognizing patterns. Therefore, we need to see what was recorded, so please don't hide the signals with fancy features. Toys are not helpful in our world, they can even be dangerous."- $\mathrm{C} 03$

Clinicians want to see the raw data in order to be able to make the correct interpretation of the events. Annotations, in the form of an additional number, graph, or mark, can assist the clinician, but not replace the actual data. Even physicians, who have little time, wanted to see the raw data. Today, technicians manually select pieces in the recordings (e.g. by making screenshots) that might be interesting for the physician. Still, the physician often opens the recordings to have a look himself.

During the observations, clinicians showed us how filters and algorithms can turn a meaningless signal (electrical noise that was caused by a loose connector) into a credible statement on sleep (an apnea or snoring). Filters are necessary to eliminate power line interference or to adjust the image to the situation. Every patient is different, e.g. some people have a thicker skull, and that might affect the signal strength and quality. In that case, the clinician has a broad range of features to get a clear image on the signals. We recommend making the raw data available to the clinician as a good starting point, on which advanced techniques can be applied, with care, if required.

\section{Add context to the data}

The sleep clinician analyzes the data by comparing the signals to find patterns or deviations in the recorded data that can indicate the presence of a sleep related disorder. To do so, 
additional context is required to put the recordings in perspective. One of the observed clinicians explains why:

"Often there is an overlap of problems (a decrease in oxygen saturation, waking up, a leg movement, breathing stops, ...). Then, context provides clarity about the cause and the effect. Often, it is a chicken-and-egg problem."-C01

In a hospital-based study, the clinicians have a lot of contact with the patient, as we discussed earlier. This also means that they can ask questions and let patients fill out some questionnaires during their stay at the hospital. These answers are not directly analyzed, but they do reveal a lot of information on the patient's sleep hygiene, routines, and emotions. So we saw that the communication with the patient is not only beneficial for compliance, but also surrounds the recordings with context, which can help the clinician during the analysis of the data.

Also, hospital-based sleep centers are equipped with cameras and microphones that record the patient during the night. In a monitoring room, a clinician guards the study overnight and notes striking events for the clinician who will analyze the signals. This information is very valuable because it provides the required context for the clinician to make a correct interpretation of the recorded data.

Sleep monitoring tools that enable the clinician to gather additional information can overcome the lack of video recordings or in person monitoring overnight, because they put the recorded signals in context. Of course, questions or questionnaires can also be asked through the tool. And just like the clinician asks questions while explaining the patient what is happening, the tool can do this as well. It could also be valuable to involve partners or parents to provide extra context information, but it should not disturb their or the patient's sleep (e.g. with auditory or visual feedback).

\section{E. Make data transferrable}

Sleep research does not stand on its own: a doctor refers the patient to the sleep center and gets feedback afterwards. Also, sleep research is a multidisciplinary activity bringing together multiple clinicians. If the clinician can't find the cause of the observed disruptions, specialists from diverse disciplines can be consulted (e.g. pneumology, neurology, psychology, ...). If the sleep monitoring tools allow data exchange through industry standards (e.g. using $\mathrm{EDF}+$ ), specialists can take a look at the recorded data from their own perspective, in order to make a correct diagnosis. The lack of standardized data formats forces the sleep clinician to use workarounds or even render communication between specialists impossible. During our observations, we saw clinicians emailing screenshots to each other because the software didn't allow an easy export of the data.

Capturing as much signals as possible, in the finest detail as possible, limits the sleep center as well. Redundant signals can be useful, to prevent data loss when sensor connectivity breaks during the night, but should be implemented with care. Most signals don't require great detail, so we recommend that it should be possible to limit the number of signals and the size of the recordings, to allow maximum flexibility for the clinician to share the data with other sleep professionals. One of the sleep centers where we performed our observations recently reduced the amount of data they record in a sleep study. A clinician justifies the decision to leave out a number of signals:

"Big data is nonsense, because it's not transportable. Besides, we are obliged by law to store all recorded data for 10 years, so it easily gets expensive."- $\mathrm{C} 08$

We saw this sleep center recording $100 \mathrm{MB}$ of signal data per patient per night, while the other observed sleep centers record up to $2,5 \mathrm{~GB}$ of signal data and $25 \mathrm{~GB}$ of video data per patient per night. The first center managed to share data and ask opinions of others, the latter could only consult the recorded data on their local computers. Both centers delivered similar reports to the referring GP.

\section{DISCUSSION}

Clinical sleep research is searching for solutions to deal with increasing demand for sleep studies. In the quantified self movement on the other hand, sleep monitoring tools are being used for data collection at home, but these tools do not pass clinical validation and thus do not find entrance in the hospital. We discuss how the same sleep monitoring tool could provide answers for both the sleep clinician and the quantified selfer, based on our findings.

\section{A. Tools for clinicians in (ambulatory) sleep research}

In traditional sleep research, the patient goes to his doctor with some complaints (e.g. feeling tired or snoring). The doctor examines the patient and can refer the patient to the hospital to perform a full PSG for one night if he presumes the patient suffers from a sleep related disorder. In the hospital, the sleep center performs a PSG to reveal the sleep related disorder(s). To do this, the hospital collects as much data as possible (e.g. an EEG, ECG, EMG, ...). The clinician applies a lot of sensors on the patient, and the patient sleeps in the hospital. After the data collection, a specialist analyzes the recorded data of the whole night by examining and comparing signals in a 30 second timeframe (for EEG) or 5 minute time frame for other signals. After the symptoms are identified, the sleep clinician or specialists can look for underlying disorders. The analysis is a time consuming process as a lot of different signals need to be examined, and the puzzle can have multiple plausible solutions. At the end, the clinician sends a report with the findings to the doctor. The doctor can then start a treatment if required.

The sleep center has a broad focus to make sure that the expression of the sleep related disorder is recorded. So the hospital uses a lot of sensors, making it more expensive and obtrusive for the patient. On the other hand, the duration of a PSG is mostly limited to one night, because the sleep center is limited by the number of available beds in the hospital. These recordings should therefore be seen as a snapshot. Factors that have in influence on sleep in the long-term fly under the radar and are difficult to examine. Balancing the scope, cost, and duration of a sleep study is a difficult exercise.

Ambulatory sleep research follows a similar procedure. The main difference is that the patient sleeps at home. Using less 
obtrusive sensors, it would even be possible to extend these kinds of measurements for a longer period of time. The sleep center would now be limited by the amount of sensors and available time for analysis, and not by the amount of beds in the center. The actual cost for these kinds of measurements will also be lower. For the patient however, all depends on reimbursement policies. E.g. in Belgium, unguarded sleep studies are no longer reimbursed to prevent abuses of the reimbursement systems, so it becomes too expensive for the patient and isn't performed anymore.

When we can provide the sleep clinician with tools that fit in the existing hospital equipment and procedures, these tools can be used both in the hospital and at home. The tool does not limit the clinician, so he can choose what is best depending on the needs for the patient. In the hospital, new tools can augment - not replace - existing techniques like PSG, and in ambulatory sleep studies, long-term measurements become feasible. For the patient, this can also be beneficial because he can sleep at home while the clinician still receives reliable data as if the measurements were done in the hospital.

While sleeping at home, the patient is in need for a good explanation, to make sure that all measurements happen correctly, and that the patient accepts what is happening (compliance). In the hospital, this explanation should not replace the clinician, but can be a helping hand for the clinician.

After the measurements are done, the clinician should have access to the raw data in a standardized format. Then, the recorded data can be seamlessly integrated in the current workflow, and gives the clinicians a good starting point to make medical decisions. If this data can be enriched with context, the clinician has the required information to analyze the recorded signals and the patient can be confident that no valuable information is lost, even when there is less contact with the clinician e.g. in an ambulatory study. And the clinician should be able to share the data with colleagues in other specialties or hospitals, to make a thorough analysis. After all, sleep research is a multidisciplinary activity.

Tools that are designed with these recommendations in mind give clinicians maximum flexibility to perform sleep studies as required, in a targeted, meaningful, and accountable way. The patient from his side gets involved in the process, and receives the care he needs.

\section{B. Tools for the quantified selfer}

In the quantified self movement, measurements are selfinitiated. A person chooses to track certain aspects of his life, e.g. his night's rest, to build up self-knowledge. Here, the sleeper is not a patient, but acts as client for the system. He decides to make use of certain tools or services. The sleeper has full control over the measurements, but also stands alone to make an interpretation of the recorded data. In practice, we see that this step is difficult because sleep patterns are complex. To understand the data, it would be good if the patient could consult a trained professional e.g. a clinician in a sleep center.

Suppose a sleep related disorder is present, then the quantified selfer could notice a distorted pattern. The sleep a.

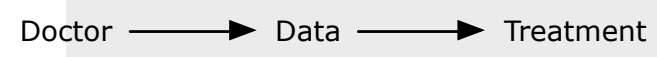

b.

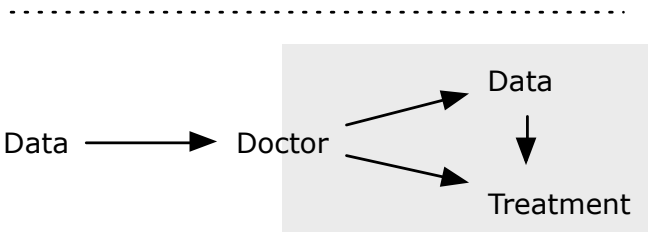

Fig. 2. In the flow of a traditional sleep study (a), the doctor has almost full control (illustrated by the grey rectangle). In a sleep study expanded by quantified self (b), the patient can help the doctor while keeping some control over the data collection.

monitoring tool could also assist the user in deciding whether there is a need for a medical intervention. The client can now address his doctor in a much more targeted way, and become a patient. The doctor could make use of the available data that the patient recorded himself, as illustrated in Fig. 2. The data that was collected by the quantified selfer, on the sleeper's initiative, can now serve the doctor and the sleep clinician in the sleep center. As we see in Fig. 2., quantified self does not replace the doctor or his tasks (the grey area). The doctor can still begin with data collection as he would have done in a traditional sleep study and then treat the patient. But he has also the possibility to use the data that was collected by the quantified selfer.

If the sleep monitoring tool can fit in existing hospital procedures and gives the clinician access to the raw data enriched with context, the output can be used by the clinician right away, without the need to perform the same measurements over again. When standardized data formats are being used, the clinician can share the data with colleagues for a thorough analysis. As well for the clinician as for the quantified selfer, a good explanation is required to make sure the measurements happen correctly, and it can help the sleeper to make sense of the complex sleep patterns. In the same way, the sleeper can play a role in data collection during or after treatment. We can think of interesting feedback loops between doctors and patients to have a more extensive follow-up than a snapshot once in a while using expensive techniques like PSG nowadays .

\section{CONCLUSION}

The transition from the hospital to ambulatory care is going on right now. If we look at past evolutions, it is conceivable that at some point in time people will monitor sleep themselves. For example a thermometer, once an instrument that was used only by scientists, is now present in almost any household. In the same way, sleep monitoring tools could become a widespread instrument for people to look at their own health, after which they can consult a doctor for further examination or treatment. 
Quantified self can have an added value to this evolution in medical procedures, to enrich the data on which doctors base their diagnosis and further decisions. This data collection can be installed before, during, or after current practices, as a way to augment and expand screening, treatment, and follow-up.

In this evolution of medical procedures, a lot of challenges will come up. These challenges are only to a small extent technical, as the technology to measure data at home and transfer it in a secure way to the hospital is already available today. However, on social, political and economical level, the road is still long. As we saw during our observations, reimbursement policies have a major impact on which tools and procedures are being used in the hospital.

Using tools that make sense from a medical point of view, meaning the tools or the output they produce can be used by clinicians because they fit the workflow in the hospital and let them access raw data, people are able to have or take control over their data. We saw that patient empowerment can have benefits for everyone involved in sleep studies, as it is beneficial for compliance reasons. At the same time, the patient can enrich the data with context, which the clinicians can use and share among colleagues to make a correct analysis. These sleep monitoring tools that can transcend the borders between the home and the hospital, and between the people and doctors, leading to a thorough and cost-efficient health care.

\section{ACKNOWLEDGMENTS}

The research described is part of the NXT_SLEEP project co-founded by iMinds (Interdisciplinary Institute for Technology), a research institute founded by the Flemish Government. Companies and organizations involved in the project are IMEC, Sensotiss, University Hospital Antwerp, Custom8, Fifthplay, and NXP Semiconductors, with project support of IWT for the latter three. Academic partners are VUB-SMIT, KU Leuven-STADIUS, and KU Leuven-CUO.

\section{REFERENCES}

[1] American Academy of Sleep Medicine. International classification of sleep disorders, revised: Diagnostic and coding manual. American Academy of Sleep Medicine, 2001.

[2] Aliakseyeu, D., Du, J., Zwartkruis-Pelgrim, E., and Subramanian, S. Exploring Interaction Strategies in the Context of Sleep. Proceedings of the 13th IFIP TC 13 International Conference on Human-computer Interaction - Volume Part III, Springer-Verlag (2011), 19-36.

[3] Bardram, J.E., Frost, M., Szántó, K., and Marcu, G. The MONARCA self-assessment system: a persuasive personal monitoring system for bipolar patients. Proceedings of the 2nd ACM SIGHIT International Health Informatics Symposium, ACM (2012), 21-30.

[4] Bauer,J.S.,Consolvo,S.,Greenstein,B.,etal.ShutEye: Encouraging Awareness of Healthy Sleep Recommendations with a Mobile, Peripheral Display. Proceedings of the SIGCHI Conference on Human Factors in Computing Systems, ACM (2012), 1401- 1410.

[5] Beyer,H.andHoltzblatt,K.ContextualDesign: Defining Customercentered Systems. Morgan Kaufmann, 1998.

[6] Biggs,J.RunningOnEmpty:WakeMateFindsOut What Happens When $\begin{array}{llll}\text { Partners } & \text { Break } & \text { Up. } & \text { TechCrunch. }\end{array}$ http://techcrunch.com/2012/06/28/running-on-empty- wakemate-findsout-what-happens-when-partners- break-up/.

[7] Choe,E.K.,Consolvo,S.,Watson,N.F.,andKientz, J.A. Opportunities for Computing Technologies to Support Healthy Sleep Behaviors.
Proceedings of the SIGCHI Conference on Human Factors in Computing Systems, ACM (2011), 3053-3062.

[8] Choe,E.K.DesignofPersuasiveTechnologiesfor Healthy Sleep Behavior. Proceedings of the 13th International Conference on Ubiquitous Computing, ACM (2011), 507-510.

[9] Dolen,B.Exclusive:sleepcoachcompanyZeois shutting down. MobiHealthNews. http://mobihealthnews.com/20772/exclusive-sleepcoach-company-zeo-is-shutting-down/.

[10] Gartenberg,D.,Thornton,R.,Masood,M.,Pfannenstiel, D., Taylor, D., and Parasuraman, R. Collecting Health- related Data on the Smart Phone: Mental Models, Cost of Collection, and Perceived Benefit of Feedback. Personal Ubiquitous Comput. 17, 3 (2013), 561-570.

[11] Han, J., Chong, J.Y., and Kim, S. SNORES: Towards a Less-intrusive Home Sleep Monitoring System Using Wireless Sensor Networks. Proceedings of the 7th ACM Conference on Embedded Networked Sensor Systems, ACM (2009), 339-340.

[12] Kelly, J.M., Strecker, R.E., and Bianchi, M.T. Recent Developments in Home Sleep-Monitoring Devices. International Scholarly Research Notices 2012, (2012).

[13] Koreshoff, T.L., Robertson, T., and Leong, T.W. Internet of Things: A Review of Literature and Products. Proceedings of the 25th Australian Computer-Human Interaction Conference: Augmentation, Application, Innovation, Collaboration, ACM (2013), 335-344.

[14] Kushida, C.A., Chang, A., Gadkary, C., Guilleminault, C., Carrillo, O., and Dement, W.C. Comparison of actigraphic, polysomnographic, and subjective assessment of sleep parameters in sleep-disordered patients. Sleep Medicine 2, 5 (2001), 389-396.

[15] Lawson, S., Jamison-Powell, S., Garbett, A., et al. Validating a Mobile Phone Application for the Everyday, Unobtrusive, Objective Measurement of Sleep. Proceedings of the SIGCHI Conference on Human Factors in Computing Systems, ACM (2013), 2497-2506.

[16] Li, I., Dey, A.K., and Forlizzi, J. Understanding My Data, Myself: Supporting Self-reflection with Ubicomp Technologies. Proceedings of the 13th International Conference on Ubiquitous Computing, ACM (2011), 405-414.

[17] Paalasmaa, J., Waris, M., Toivonen, H., Leppakorpi, L., and Partinen, M. Unobtrusive online monitoring of sleep at home. 2012 Annual International Conference of the IEEE Engineering in Medicine and Biology Society (EMBC), (2012), 3784-3788.

[18] Pirzadeh, A., He, L., and Stolterman, E. Personal Informatics and Reflection: A Critical Examination of the Nature of Reflection. CHI '13 Extended Abstracts on Human Factors in Computing Systems, ACM (2013), 1979-1988.

[19] Rivas-Echeverría, C., Acosta, E., Rivas-Echeverría, F., Molina, L., González, S., and Sánchez, R. Features and Applications of an Information System Developed for a Sleep Clinic. Proceedings of the 9th WSEAS International Conference on Computational Intelligence, Man-machine Systems and Cybernetics, World Scientific and Engineering Academy and Society (WSEAS) (2010), 209-215.

[20] Shambroom, J.R., Fábregas, S.E., and Johnstone, J. Validation of an automated wireless system to monitor sleep in healthy adults. Journal of Sleep Research 21, 2 (2012), 221-230.

[21] Verbraecken, J., Raymann, R., and Eijsvogel, M. Ambulante P(S)G. In Leerboek slaap en slaapstoornissen. Acco, 2013, 167-172.

[22] Wright, A. Patient, Heal Thyself. Commun. ACM 56, 8 (2013), 16-18.

[23] Chen, Y., Tang, C., Cheng, K., and Park, S.Y. Bridging clinical and nonclinical health practices: opportunities and challenges. CHI '12 Extended Abstracts on Human Factors in Computing Systems, ACM (2012), 2723-2726.

[24] Zhang, J., Zhang, Q., Wang, Y., and Qiu, C. A Real- time Autoadjustable Smart Pillow System for Sleep Apnea Detection and Treatment. Proceedings of the 12th International Conference on Information Processing in Sensor Networks, ACM (2013), 179-190.

[25] Zhao, W., Wang, X., and Wang, Y. Automated Sleep Quality Measurement Using EEG Signal: First Step Towards a Domain Specific Music Recommendation System. Proceedings of the International Conference on Multimedia, ACM (2010), 1079-10. 